\title{
Horizontal and vertical tree community structure in a lowland Atlantic Rain Forest, Southeastern Brazil
}

\author{
FREDERICO AUGUSTO G. GUILHERME ${ }^{1}$, L. PATRÍCIA C. MORELLATO ${ }^{1}$ and MARCO A. ASSIS ${ }^{1,2}$
}

(received: April 2, 2003; accepted: August 12, 2004)

\begin{abstract}
Horizontal and vertical tree community structure in a lowland Atlantic Rain Forest, Southeastern Brazil). The horizontal and vertical tree community structure in a lowland Atlantic Rain Forest was investigated through a phytosociological survey in two 0.99 ha plots in the Intervales State Park, São Paulo State. All trees $\geq 5 \mathrm{~cm}$ diameter at breast height were recorded. 3,078 individuals belonging to 172 species were identified and recorded. The Shannon diversity index was H' $=3.85$ nat.ind.$^{-1}$. The Myrtaceae family showed the greatest floristic richness ( 38 species) and the highest density (745 individuals) in the stand. Euterpe edulis Mart. had the highest importance value (33.98\%) accounting for $21.8 \%$ of all individuals recorded. The quantitative similarity index was higher than the qualitative index, showing little structural variation between plots. However, the large number of uncommon species resulted in pronounced floristic differences. A detrended correspondence analysis (DCA) generated three arbitrary vertical strata. Stratum A ( $\geq 26 \mathrm{~m})$, where Sloanea guianensis (Aubl.) Benth. and Virola bicuhyba (Schott. ex A.DC.) Warb. were predominant showed the lowest density. Stratum B $(8 \mathrm{~m}<\mathrm{h}<26 \mathrm{~m})$ had the greatest richness and diversity, and stratum C $(\leq 8 \mathrm{~m})$ showed the highest density. Euterpe edulis, Guapira opposita (Vell.) Reitz, Garcinia gardneriana (Planch. \& Triana) Zappi, and Eugenia mosenii (Kausel) Sobral were abundant in strata B and C. The occurrence of strata in tropical forests is discussed and we recommend the use of DCA for others studies of the vertical distribution of tropical forest tree communities.
\end{abstract}

Key words - diversity, multivariate analysis, phytosociology, tree stratification, tropical forest

RESUMO - (Estruturas horizontal e vertical do componente arbóreo em floresta atlântica baixo-montana no sudeste do Brasil). A estrutura horizontal e vertical do componente arbóreo foi investigada em um trecho de Floresta Atlântica baixo-montana através de um levantamento fitossociológico em dois blocos amostrais de 0,99 ha cada no Parque Estadual Intervales. Todos os indivíduos com DAP $\geq 5 \mathrm{~cm}$ foram registrados. Foram amostrados 3.078 indivíduos distribuídos em 172 espécies. O índice de diversidade de Shannon foi de H' $=3,85$ nat.ind. ${ }^{-1}$. A família Myrtaceae se destacou tanto em número de espécies (38) quanto em número de indivíduos (745) no levantamento. Euterpe edulis Mart. teve o maior valor de importância (33,98\%), abrangendo $21,8 \%$ do total de indivíduos registrados. O índice de similaridade quantitativo foi maior do que o qualitativo, mostrando pouca variação estrutural entre os blocos amostrais, mas a grande quantidade de espécies pouco abundantes, resultou em pronunciadas diferenças florísticas entre eles. Uma análise de correspondência retificada (DCA) gerou três estratos verticais arbitrários. O estrato A ( $\geq 26 \mathrm{~m}$ ) teve a menor densidade e foi bem representado pelas espécies Sloanea guianensis (Aubl.) Benth. e Virola bicuhyba (Schott. ex A.DC.) Warb. O estrato B $(8 \mathrm{~m}<\mathrm{h}<26 \mathrm{~m})$ mostrou a maior riqueza e diversidade florística, e o estrato C $(\leq 8 \mathrm{~m})$ a maior densidade. Euterpe edulis, Guapira opposita (Vell.) Reitz, Garcinia gardneriana (Planch. \& Triana) Zappi e Eugenia mosenii (Kausel) Sobral foram bem representadas nos estratos B e C da floresta. A existência de estratos verticais em florestas tropicais é discutida, recomendando-se o uso da DCA para estudos da estratificação vertical em outras florestas tropicais.

Palavras-chave - análise multivariada, diversidade, estratificação, fitossociologia, floresta tropical

\section{Introduction}

The Atlantic Rain Forest sensu stricto (Oliveira Filho \& Fontes 2000) originally covered an area of 1.2 million $\mathrm{km}^{2}$ along the Eastern coastline of Brazil, but today it is reduced to approximately $7.5 \%$ of its original extent (Myers et al. 2000). This biome presents one of

\footnotetext{
1. Universidade Estadual Paulista, Instituto de Biociências, Departamento de Botânica, Caixa Postal 199, 13506-900 Rio Claro, SP, Brasil.

2. Corresponding author: massis@rc.unesp.br
}

the greatest species richness as well as high levels of plant and animal endemisms (Mori et al. 1981, Leitão Filho 1994). These characteristics put the Atlantic Rain Forest among the eight most important 'hotspots' for biodiversity conservation (Myers et al. 2000).

The largest and most preserved remnants of Atlantic Rain Forest are located nearby the most developed coastal urban centers, particularly in the States of São Paulo and Paraná, Southeastern Brazil, due to the irregular landscapes of Serra do Mar and Serra de Paranapiacaba (Leitão Filho 1994). For instance, the Intervales State Park, located in the Vale do Ribeira 
region in the south of the São Paulo State, the Park was granted the status of Preservation Unit due to its extreme ecological importance. The Intervales, along with other Preservation Units, makes up for the largest continuous area of Atlantic Rain Forest hosting a biodiversity which has not yet been thoroughly studied (Dietz 2001).

Although several studies have been carried out with regard to the structure of the vegetation of the Atlantic Rain Forest, specially in the São Paulo State (Silva \& Leitão Filho 1982, Mantovani et al. 1990, Leitão Filho 1993, Melo \& Mantovani 1994, Melo et al. 1998, Oliveira et al. 2001, Sztutman \& Rodrigues 2002, among others), the data available remain insufficient for the understanding of abundance, distribution, richness, and diversity of species. Besides the lack of data, the floristic and structure of such vegetations are rather complex, varying according to edaphic-climatic and geomorphological characteristics of each region (Leitão Filho 1987, Mantovani 1998, Ivanauskas et al. 2000, Oliveira Filho \& Fontes 2000). Another aspect of the Atlantic Rain Forest which has not been thoroughly investigated so far is the characterization of the vertical structure of tree community. According to Richards (1996), tropical forests have vertical stratification. These strata are composed by several plant species with different capabilities of occupation resulting from an adaptative response to the different conditions of light. This interaction may also justify the great diversity present in more structurally complex ecosystems. Considering that existing methods to quantify the vertical structure of forests are defined arbitrarily (Latham et al. 1998) making them a controversial issue, and that the strata in tropical forests are much less defined than those in temperate forests (Terborgh 1992), studies of stratification or of the vertical structure of the canopy can offer insights to a better understanding of the natural history of the tropics. Therefore, these studies may incentive further discussions about the evolution of the types of dispersion (Roth 1987) and the distribution of the epiphytic component along a vertical gradient (Kersten \& Silva 2001, 2002), among others.

This study aimed (a) to describe the horizontal structure of the tree community of a lowland Atlantic Rain Forest and to compare it with other studies carried out in this vegetation; (b) to investigate the vertical structure of such community, assessing how the stratification or the vertical structure of canopy can be defined along a section of the forest and in which ways the composition and importance of species in each stratum can vary, based on the most extensive survey $(\sim 2$ ha) carried out up today.

\section{Material and methods}

Study site - This study was conducted in the Intervales State Park (Parque Estadual Intervales - PEI), Base Saibadela (24'14' S and $48^{\circ} 04^{\prime}$ 'W), located in the Serra de Paranapiacaba, municipality of Sete Barras, southern São Paulo State, southeast Brazil. The PEI stretches along an area of approximately 48,000 ha and it is surrounded by three other Preservation Units (Parque Estadual Carlos Botelho, Estação Ecológica do Xitué and Parque Estadual Turístico do Alto Ribeira), accounting for 200,000 ha of preserved forest. It is one of the most important areas for the preservation of the Atlantic Forest Biosphere Reserve (Dietz 2001).

The PEI has a very irregular landscape and the local altitude ranges from 60 to $1,100 \mathrm{~m}$. At the Base Saibadela, the altitude ranges from around 70 to $250 \mathrm{~m}$. Base Saibadela is situated on the coastal mountain chain of Serra de Paranapiacaba that presents ample erosive landscapes of mountainous aspects, formed by steep scarps and deep valleys, which represent the rim of the Atlantic Crystalline Plateau, composed mainly of granitic and gneissic rocks of high structural complexity (Almeida 1974). The climatic data registered at the study area show a wet tropical climate, with frequent rains without a period of hydric deficit and annual precipitation around $4,000 \mathrm{~mm}$, with a less rainy season from May to August and a wet season from September to April (Morellato et al. 2000). The average temperature in the coldest month (July) is $18.1^{\circ} \mathrm{C}$ and in the warmest month (January) is $25.7^{\circ} \mathrm{C}$. According to Setzer (1966) the region presents a Cfb type climate in the Köppen system - temperate without a dry season.

The study area is covered mainly by primary Atlantic Rain Forest with little anthropogenic intervention and is situated within domain of lowland Atlantic Rain Forest (sensu Oliveira Filho \& Fontes 2000). The most representative families found in the tree community are Myrtaceae, Lauraceae and Rubiaceae, and the palmito-juçara, Euterpe edulis Mart. (Arecaceae), is among the most abundant species in the area (R.J. Almeida-Scabbia, unpublished data). The epiphytes are very abundant and represented especially by several species of ferns, Orchidaceae, Bromeliaceae, and Araceae. The number of liana species is also high, with 43 species registered until the present moment (V.B. Zipparro et al., unpublished data). On the foothills and in the less drained plains, due to the proximity to the water courses, clumps of Aphelandra liboniana Linden ex Hook. (Acanthaceae) and Calathea spp. (Marantaceae) cover extensive areas of the forest understorey.

Vegetation structure - The sampling was conducted from October 2000 to November 2001. Two sample plots were established for the analysis of the tree component structure. One was established in low and another in midland topographic conditions. Each was composed of 44 continuous $15 \times 15 \mathrm{~m}$ quadrats, totaling a 0.99 ha $(60 \times 165 \mathrm{~m})$ area per topographic condition. The plots were located 1,500 m 
away from each other in the lowland Atlantic Rain Forest.

Plot 1 is situated perpendicular and ca. $25 \mathrm{~m}$ away from the Saibadela river margin, and was located at a mean altitude of $120 \mathrm{~m}$ (amplitude of 110-130 m), with predominance of dystrophic cambisoils. The first four quadrats are situated over the old river course, since there is a great number of pebbles in a dystrophic neosoils. As one moves away from the river, the capacity of soil drainage generally increases, except in one point in the center of Plot 1 were the water table rises permanently above the soil surface over the dystrophic gleisoils. Plot 2 is situated in an area of low altitude, ranging from 86 to $97 \mathrm{~m}$, far from the river, in a well drained dystrophic cambisoil. In Plot 1, there has been human interference since 1998 , such as clandestine exploration of the palm tree palmitojuçara, which stems still stood in the soil after being partly cut.

All tree individuals with $\mathrm{DBH} \geq 5 \mathrm{~cm}$, including the palms and the ferns, were sampled, labeled and measured at breast height. The height of all individuals was estimated with the use of a $2 \mathrm{~m}$ rod. Considering the great number of palms that had been cut down in Plot $1(n=63)$, they were included in the same group with living Euterpe edulis for the assessment of horizontal structure. The vouchers of all sampled individuals are deposited at the Herbario Rioclarense (HRCB). All species in this study were grouped according to the Angiosperm Phylogeny Group (APG 2003), except the Cyatheaceae family. Data analysis - The phytosociological parameters calculed for each sample plot were: species density, frequency and relative dominance, and value of importance (VI) (MuellerDombois \& Ellenberg 1974); the Shannon diversity index (H') and the Pielou equability index (J') (Brower \& Zar 1984). Calculations were made with the use the software FITOPAC (Shepherd 1994).

Detrended correspondence analysis (DCA), which allows an indirect analysis of gradients (Hill \& Gauch 1980), was used to verify the abundance of species in each quadrat between the plots. An abundance matrix of species was generated and expressed by the number of individuals from each taxon by plot. Taxa with less than 13 individuals were excluded, leaving a total 48 species in the analysis. The less abundant species contribute very little to the analysis and only increase the number of calculations to be made (Causton 1988). The Jaccard (qualitative) and the Morisita (quantitative) similarity indexes were used to analyze the floristic and structural similarities between the two sample plots (Krebs 1999). To characterize the vertical structure of the forest, DCA analysis was also used based on a matrix of the number of individuals in each taxon by height classes. The height records were grouped in class intervals of one meter. The analysis points simultaneously to the number of individuals per height class, relating the highest occurrence of species in each class. The resulting diagram displays a distribution of height classes split into arbitrary groups and generates the vertical distribution of the stand. The palms that had been cut were excluded from this analysis. The phytosociological parameters were separately calculated for the components of each stratum identified in the analysis of vertical distribution. The distribution of abundance of the main species was assessed in each stratum in terms of the total number of individuals in the stand with the use of quisquare test $\left(\chi^{2}\right)$ with Yates correction (Zar 1996), in order to verify whether any tree species was significantly associated to a given stratum. In this study the terms stratification and stratum are used in accordance with Richards' proposal (1996).

\section{Results}

Horizontal structure - We recorded 3,078 individuals of 172 species distributed in 102 genera and 51 botanic families. The estimated total density was $1,554.6$ ind.ha $^{-1}$ and the estimated basal area was $34.64 \mathrm{~m}^{2} \cdot \mathrm{ha}^{-1}$. The Shannon diversity index was 3.85 nat.ind. $^{-1}$ and the equability index was 0.75 .

The Euterpe edulis palm was the most abundant species adding $21.8 \%$ of the total recorded individuals. It had the highest density, frequency and value of importance (VI) (table 1). Sloanea guianensis, Bathysa australis, Virola bicuhyba and Alchornea triplinervia high VI values were due to their elevated basal areas. Guapira opposita, Eugenia mosenii, Garcinia gardneriana, Marlierea obscura and Psychotria suterella high VI values were due to their high abundance and frequency. These 10 species accounted for $39.6 \%$ of the total VI (table 1). The most abundant families represented $82.9 \%$ of the total number of individuals sampled (figure 1) wherein Myrtaceae alone contributed with $24.2 \%$ (745 individuals). Myrtaceae was the family with the highest number of species (38), followed by Fabaceae (19), Rubiaceae (13), Lauraceae (10), Sapotaceae (7) and Euphorbiaceae (6). Comparison of the floristic structure and similarities between the sample plots - Plot 1 presented lower density of trees and higher basal area (1,495 ind.ha ${ }^{-1}$ and $\left.35.6 \mathrm{~m}^{2} . \mathrm{ha}^{-1}\right)$ than Plot $2\left(1,614\right.$ ind.ha ${ }^{-1}$ and $\left.33.7 \mathrm{~m}^{2} \cdot \mathrm{ha}^{-1}\right)$. Nine out of the twelve species with highest VI in the stand were the most representative in both plots, only showing variation in the VI ranking (table 2). Bathysa australis and Marlierea tomentosa presented higher density in Plot 1, whereas Euterpe edulis, Garcinia gardneriana and Psychotria suterella had higher density in Plot 2. Despite present a similar density in both plots, individuals of Virola bicuhyba reached larger sizes in Plot 1, resulting in a greater basal area.

When analyzing the DCA diagram in regard to species abundance (figure 2), short gradients were found for the first two axes (8.29 and 9.09), with eigenvalues 
Table 1. Phytosociological descriptors of tree species with $\mathrm{DBH} \geq 5 \mathrm{~cm}$ sampled in 1.98 ha of lowland Atlantic Rain Forest, Intervales State Park, Southeastern Brazil. NI = number of individuals; $\mathrm{BA}=$ basal area $\left(\mathrm{m}^{2}\right) ; \mathrm{Q}=$ number of quadrats where it occurs; $\mathrm{RD}=$ relative density $(\%) ; \mathrm{RDo}=$ relative dominance $(\%) ; \mathrm{RF}=$ relative frequency $(\%) ; \mathrm{VI}=$ value of importance $(\%)$. * 63 out of 671 individuals of $E$. edulis had been cut by clandestine collectors. $\dagger:$ species exclusively found in Plot 1 ; $\ddagger$ : species exclusively found in Plot 20.

\begin{tabular}{|c|c|c|c|c|c|c|c|c|}
\hline Family & Species & NI & $\mathrm{BA}$ & $\mathrm{Q}$ & $\mathrm{RD}$ & RDo & $\mathrm{RF}$ & $\mathrm{VI}$ \\
\hline Arecaceae & Euterpe edulis Mart. & $* 671$ & 4.936 & 88 & 21.8 & 7.2 & 5.0 & 33.98 \\
\hline Elaeocarpaceae & Sloanea guianensis (Aubl.) Benth. & 93 & 7.173 & 54 & 3.0 & 10.5 & 3.1 & 16.54 \\
\hline Nyctaginaceae & Guapira opposita (Vell.) Reitz & 172 & 2.044 & 71 & 5.6 & 3.0 & 4.0 & 12.59 \\
\hline Rubiaceae & Bathysa australis (A.St.-Hil.) Benth. \& Hook. f. & 100 & 2.777 & 51 & 3.3 & 4.1 & 2.9 & 10.19 \\
\hline Myrtaceae & Eugenia mosenii (Kausel) Sobral & 114 & 0.938 & 64 & 3.7 & 1.4 & 3.6 & 8.70 \\
\hline Clusiaceae & Garcinia gardneriana (Planch. \& Triana) Zappi & 117 & 0.998 & 53 & 3.8 & 1.5 & 3.0 & 8.26 \\
\hline Myrtaceae & Marlierea obscura O.Berg & 94 & 1.115 & 60 & 3.1 & 1.6 & 3.4 & 8.08 \\
\hline Myristicaceae & Virola bicuhyba (Schott. ex A.DC.) Warb. & 24 & 3.604 & 20 & 0.8 & 5.3 & 1.1 & 7.17 \\
\hline Euphorbiaceae & Alchornea triplinervia (Spreng.) Müll.Arg. & 25 & 3.329 & 23 & 0.8 & 4.9 & 1.3 & 6.97 \\
\hline Rubiaceae & Psychotria suterella Müll.Arg. & 95 & 0.411 & 48 & 3.1 & 0.6 & 2.7 & 6.40 \\
\hline Myrtaceae & Marlierea tomentosa Cambess. & 78 & 0.516 & 44 & 2.5 & 0.8 & 2.5 & 5.78 \\
\hline Monimiaceae & Mollinedia schottiana (Spreng.) Perkins & 66 & 0.322 & 46 & 2.1 & 0.5 & 2.6 & 5.22 \\
\hline Olacaceae & Tetrastylidium grandifolium (Baill.) Sleumer & 46 & 1.081 & 32 & 1.5 & 1.6 & 1.8 & 4.88 \\
\hline Myrtaceae & Calycorectes australis D.Legrand & 41 & 1.105 & 31 & 1.3 & 1.6 & 1.8 & 4.70 \\
\hline Phyllanthaceae & Hyeronima alchorneoides Allemão & 17 & 2.136 & 15 & 0.6 & 3.1 & 0.9 & 4.52 \\
\hline Myrtaceae & Eugenia riedeliana O.Berg & 34 & 1.134 & 27 & 1.1 & 1.7 & 1.5 & 4.29 \\
\hline Lauraceae & Ocotea elegans $\mathrm{Mez}$ & 18 & 1.743 & 16 & 0.6 & 2.5 & 1.0 & 4.09 \\
\hline Cyatheaceae & Cyathea phalerata Mart. & 54 & 0.286 & 31 & 1.8 & 0.4 & 1.8 & 3.93 \\
\hline Myrtaceae & Eugenia cuprea (O.Berg) Nied. & 51 & 0.221 & 34 & 1.7 & 0.3 & 1.9 & 3.91 \\
\hline Myrtaceae & Eugenia neoglomerata Sobral & 45 & 0.284 & 35 & 1.5 & 0.4 & 2.0 & 3.86 \\
\hline Myrtaceae & Eugenia oblongata O.Berg & 43 & 0.438 & 31 & 1.4 & 0.6 & 1.8 & 3.79 \\
\hline Rubiaceae & Ixora burchelliana Müll.Arg. & 47 & 0.160 & 34 & 1.5 & 0.2 & 1.9 & 3.69 \\
\hline Fabaceae & Swartzia flaemingii Raddi & 23 & 1.187 & 21 & 0.8 & 1.7 & 1.2 & 3.67 \\
\hline Cyatheaceae & $\begin{array}{l}\text { Alsophila sternbergii (Pohl ex Sternb.) } \\
\text { D.S.Conant } \dagger\end{array}$ & 64 & 0.604 & 10 & 2.1 & 0.9 & 0.6 & 3.53 \\
\hline Fabaceae & Pterocarpus rohrii Vahl & 21 & 1.131 & 18 & 0.7 & 1.7 & 1.0 & 3.35 \\
\hline Lauraceae & Ocotea teleiandra (Meisn.) Mez & 39 & 0.210 & 30 & 1.3 & 0.3 & 1.7 & 3.27 \\
\hline Myrtaceae & Calycorectes acutatus (Miq.) Toledo & 29 & 0.750 & 20 & 0.9 & 1.1 & 1.1 & 3.17 \\
\hline Vochysiaceae & Callisthene cf. dryadum A.P.Duarte & 6 & 1.638 & 6 & 0.2 & 2.4 & 0.3 & 2.92 \\
\hline Urticaceae & Pourouma guianensis Aubl. & 22 & 0.714 & 19 & 0.7 & 1.0 & 1.1 & 2.83 \\
\hline Lecythidaceae & Cariniana estrellensis (Raddi) Kuntze & 11 & 1.256 & 11 & 0.4 & 1.8 & 0.6 & 2.81 \\
\hline Myrtaceae & Eugenia multicostata D.Legrand & 14 & 0.955 & 14 & 0.5 & 1.4 & 0.8 & 2.64 \\
\hline Olacaceae & Heisteria silvianii Schwacke & 22 & 0.433 & 22 & 0.7 & 0.6 & 1.3 & 2.59 \\
\hline Lauraceae & Cryptocaria moschata Nees \& Mart. ex Nees & 13 & 0.892 & 13 & 0.4 & 1.3 & 0.7 & 2.46 \\
\hline Monimiaceae & Mollinedia uleana Perkins & 21 & 0.448 & 18 & 0.7 & 0.7 & 1.0 & 2.35 \\
\hline Fabaceae & Pseudopiptadenia warmingii (Harms) Bukart & 9 & 1.057 & 9 & 0.3 & 1.5 & 0.5 & 2.34 \\
\hline Bignoniaceae & Tabebuia serratifolia (Vahl) G.Nicholson & 21 & 0.414 & 18 & 0.7 & 0.6 & 1.0 & 2.31 \\
\hline Rubiaceae & Psychotria mapoureoides DC. & 18 & 0.614 & 14 & 0.6 & 0.9 & 0.8 & 2.27 \\
\hline Fabaceae & Copaifera trapezifolia Hayne & 5 & 1.154 & 5 & 0.2 & 1.7 & 0.3 & 2.13 \\
\hline Apocynaceae & Aspidosperma compactinervium Kuhlm. & 5 & 1.147 & 5 & 0.2 & 1.7 & 0.3 & 2.12 \\
\hline Myrtaceae & Eugenia bocainensis Mattos & 30 & 0.104 & 17 & 1.0 & 0.2 & 1.0 & 2.09 \\
\hline Moraceae & Ficus gomelleira Kunth \& Bouché $\dagger$ & 1 & 1.325 & 1 & 0.0 & 1.9 & 0.1 & 2.02 \\
\hline Euphorbiaceae & Aparisthmium cordatum (A.Juss.) Baill. & 27 & 0.200 & 15 & 0.9 & 0.3 & 0.9 & 2.02 \\
\hline Lauraceae & Endlicheria paniculata (Spreng.) J.F.Macbr. & 20 & 0.112 & 20 & 0.7 & 0.2 & 1.1 & 1.95 \\
\hline Myrtaceae & Gomidesia spectabilis (DC.) O.Berg & 20 & 0.128 & 18 & 0.7 & 0.2 & 1.0 & 1.86 \\
\hline Sapindaceae & Cupania oblongifolia Mart. & 13 & 0.500 & 12 & 0.4 & 0.7 & 0.7 & 1.83 \\
\hline Sapindaceae & Matayba guianensis Aubl. & 8 & 0.739 & 8 & 0.3 & 1.1 & 0.5 & 1.79 \\
\hline
\end{tabular}


continuation

\begin{tabular}{|c|c|c|c|c|c|c|c|c|}
\hline Family & Species & $\mathrm{NI}$ & BA & Q & $\mathrm{RD}$ & RDo & $\mathrm{RF}$ & VI \\
\hline Polygonaceae & Ruprechtia laxiflora Meisn. & 18 & 0.241 & 15 & 0.6 & 0.4 & 0.9 & 1.79 \\
\hline Meliaceae & Cabralea canjerana (Vell.) Mart. & 6 & 0.845 & 6 & 0.2 & 1.2 & 0.3 & 1.77 \\
\hline Myrtaceae & Calyptranthes lanceolata O.Berg & 20 & 0.058 & 17 & 0.7 & 0.1 & 1.0 & 1.70 \\
\hline Quiinaceae & Quiina glaziovii Engl. & 9 & 0.670 & 7 & 0.3 & 1.0 & 0.4 & 1.67 \\
\hline Symplocaceae & Symplocos cf. celastrinea Mart. ex Miq. & 14 & 0.373 & 11 & 0.5 & 0.5 & 0.6 & 1.62 \\
\hline Myrtaceae & Eugenia cambucarana Kiaersk. † & 13 & 0.498 & 8 & 0.4 & 0.7 & 0.5 & 1.60 \\
\hline Fabaceae & Schizolobium parahyba (Vell.) S.F. Blake & 3 & 0.890 & 3 & 0.1 & 1.3 & 0.2 & 1.56 \\
\hline Euphorbiaceae & Alchornea glandulosa Poepp. & 6 & 0.684 & 6 & 0.2 & 1.0 & 0.3 & 1.53 \\
\hline Fabaceae & Inga edulis Mart. & 15 & 0.184 & 13 & 0.5 & 0.3 & 0.7 & 1.49 \\
\hline Myrtaceae & Myrciaria floribunda (West ex Willd.) O.Berg & 11 & 0.309 & 11 & 0.4 & 0.5 & 0.6 & 1.43 \\
\hline Urticaceae & Coussapoa microcarpa (Schott) Rizzini & 8 & 0.448 & 8 & 0.3 & 0.7 & 0.5 & 1.37 \\
\hline Rutaceae & Pilocarpus pauciflorus A.St.-Hil. & 17 & 0.096 & 12 & 0.6 & 0.1 & 0.7 & 1.37 \\
\hline Sapotaceae & Pouteria venosa (Mart.) Baehni & 10 & 0.330 & 9 & 0.3 & 0.5 & 0.5 & 1.32 \\
\hline Apocynaceae & Malouetia cestroides Müll.Arg. & 10 & 0.308 & 9 & 0.3 & 0.5 & 0.5 & 1.28 \\
\hline Proteaceae & Roupala brasiliensis Klotzsch & 10 & 0.254 & 9 & 0.3 & 0.4 & 0.5 & 1.20 \\
\hline Lauraceae & Ocotea dispersa (Nees) Mez & 12 & 0.119 & 11 & 0.4 & 0.2 & 0.6 & 1.19 \\
\hline Rubiaceae & $\begin{array}{l}\text { Rudgea jasminoides subsp. jasminoides } \\
\text { (Cham.) Müll.Arg. }\end{array}$ & 14 & 0.064 & 11 & 0.5 & 0.1 & 0.6 & 1.17 \\
\hline Celastraceae & Maytenus robusta Reissek $\ddagger$ & 10 & 0.327 & 6 & 0.3 & 0.5 & 0.3 & 1.14 \\
\hline Sapotaceae & Diploon cuspidatum (Hoehne) Cronquist & 13 & 0.087 & 10 & 0.4 & 0.1 & 0.6 & 1.12 \\
\hline Myrtaceae & Plinia complanata M.L.Kawas. \& B.Holst & 13 & 0.048 & 11 & 0.4 & 0.1 & 0.6 & 1.12 \\
\hline Rubiaceae & Alseis floribunda Schott & 7 & 0.336 & 6 & 0.2 & 0.5 & 0.3 & 1.06 \\
\hline Myrtaceae & Eugenia subavenia O.Berg $\ddagger$ & 11 & 0.085 & 10 & 0.4 & 0.1 & 0.6 & 1.05 \\
\hline Fabaceae & Myrocarpus frondosus Allemão & 6 & 0.317 & 6 & 0.2 & 0.5 & 0.3 & 1.00 \\
\hline Icacinaceae & Citronella megaphylla (Miers) Howard & 9 & 0.128 & 9 & 0.3 & 0.2 & 0.5 & 0.99 \\
\hline Arecaceae & Astrocarium aculeatissimum (Schott) Burret & 10 & 0.109 & 9 & 0.3 & 0.2 & 0.5 & 0.99 \\
\hline Myrtaceae & Eugenia cerasiflora Miq. $\ddagger$ & 5 & 0.349 & 5 & 0.2 & 0.5 & 0.3 & 0.95 \\
\hline Myrtaceae & Eugenia pruinosa D.Legrand & 6 & 0.282 & 6 & 0.2 & 0.4 & 0.3 & 0.95 \\
\hline Sapotaceae & Pouteria caimito (Ruiz \& Pav.) Radlk. & 8 & 0.165 & 7 & 0.3 & 0.2 & 0.4 & 0.90 \\
\hline Rubiaceae & Alibertia myrcifolia K.Schum. & 9 & 0.043 & 9 & 0.3 & 0.1 & 0.5 & 0.86 \\
\hline Fabaceae & Dahlstedtia pentaphylla (Taub.) Burkart & 9 & 0.042 & 9 & 0.3 & 0.1 & 0.5 & 0.86 \\
\hline Annonaceae & Guatteria australis A.St.-Hill. & 9 & 0.031 & 9 & 0.3 & 0.0 & 0.5 & 0.85 \\
\hline Chrysobalanaceae & Hirtella hebeclada Moric. ex DC. & 7 & 0.123 & 7 & 0.2 & 0.2 & 0.4 & 0.80 \\
\hline Chrysobalanaceae & Parinari excelsa Sabine & 4 & 0.295 & 4 & 0.1 & 0.4 & 0.2 & 0.79 \\
\hline Myrtaceae & Myrcia macrocarpa Barb.Rodr. & 7 & 0.084 & 7 & 0.2 & 0.1 & 0.4 & 0.75 \\
\hline Myrtaceae & Eugenia neoverrucosa Sobral & 8 & 0.063 & 7 & 0.3 & 0.1 & 0.4 & 0.75 \\
\hline Myrtaceae & Eugenia beaurepairiana (Kiaersk.) D.Legrand & 6 & 0.131 & 6 & 0.2 & 0.2 & 0.3 & 0.73 \\
\hline Myrtaceae & Eugenia sp. 1 & 7 & 0.073 & 7 & 0.2 & 0.1 & 0.4 & 0.73 \\
\hline Lamiaceae & Aegiphyla sellowiana Cham. & 6 & 0.105 & 6 & 0.2 & 0.2 & 0.3 & 0.69 \\
\hline Bignoniaceae & Jacaranda cf. montana Morawetz & 4 & 0.202 & 4 & 0.1 & 0.3 & 0.2 & 0.65 \\
\hline Celastraceae & Maytenus cf. alaternoides Reissek & 6 & 0.074 & 6 & 0.2 & 0.1 & 0.3 & 0.64 \\
\hline Meliaceae & Guarea macrophylla Vahl & 6 & 0.071 & 6 & 0.2 & 0.1 & 0.3 & 0.64 \\
\hline Sapotaceae & Ecclinusa ramiflora Mart. $\dagger$ & 7 & 0.079 & 5 & 0.2 & 0.1 & 0.3 & 0.63 \\
\hline Myrtaceae & Myrcia tenuivenosa Kiaersk. & 4 & 0.182 & 4 & 0.1 & 0.3 & 0.2 & 0.62 \\
\hline Myrtaceae & Gomidesia anacardiifolia (Gardner) O.Berg & 6 & 0.044 & 6 & 0.2 & 0.1 & 0.3 & 0.60 \\
\hline Fabaceae & Machaerium sp. $\dagger$ & 1 & 0.334 & 1 & 0.0 & 0.5 & 0.1 & 0.58 \\
\hline Fabaceae & Sclerolobium denudatum Vogel $\ddagger$ & 1 & 0.334 & 1 & 0.0 & 0.5 & 0.1 & 0.58 \\
\hline Euphorbiaceae & Actinostemon concolor (Spreng.) Müll.Arg. & 6 & 0.073 & 5 & 0.2 & 0.1 & 0.3 & 0.58 \\
\hline Euphorbiaceae & Tetrorchidium rubrivenium Poepp. & 4 & 0.143 & 4 & 0.1 & 0.2 & 0.2 & 0.57 \\
\hline Myrtaceae & Eugenia melanogyna (D.Legrand) Sobral & 6 & 0.055 & 5 & 0.2 & 0.1 & 0.3 & 0.56 \\
\hline Fabaceae & Ormosia cf. arborea (Vell.) Harms & 5 & 0.060 & 5 & 0.2 & 0.1 & 0.3 & 0.53 \\
\hline Sapotaceae & Chrysophyllum inornatum Mart. † & 3 & 0.175 & 3 & 0.1 & 0.3 & 0.2 & 0.52 \\
\hline
\end{tabular}


continuation

\begin{tabular}{|c|c|c|c|c|c|c|c|c|}
\hline Family & Species & NI & $\mathrm{BA}$ & Q & $\mathrm{RD}$ & RDo & $\mathrm{RF}$ & $\mathrm{VI}$ \\
\hline Myrtaceae & Myrceugenia myrcioides (Cambess.) O.Berg & 6 & 0.023 & 5 & 0.2 & 0.0 & 0.3 & 0.51 \\
\hline Myrtaceae & Myrceugenia sp. & 4 & 0.098 & 4 & 0.1 & 0.1 & 0.2 & 0.50 \\
\hline Rubiaceae & Rudgea recurva Müll.Arg. & 5 & 0.023 & 5 & 0.2 & 0.0 & 0.3 & 0.48 \\
\hline Boraginaceae & Cordia silvestris Fresen. $†$ & 3 & 0.141 & 2 & 0.1 & 0.2 & 0.2 & 0.47 \\
\hline Fabaceae & Inga sessilis (Vell.) Mart. & 4 & 0.069 & 4 & 0.1 & 0.1 & 0.2 & 0.46 \\
\hline Rubiaceae & Posoqueria latifolia (Rudge) Roem. \& Schult. & 4 & 0.053 & 4 & 0.1 & 0.1 & 0.2 & 0.43 \\
\hline Myrtaceae & Eugenia cf.platysema O.Berg & 4 & 0.041 & 4 & 0.1 & 0.1 & 0.2 & 0.42 \\
\hline Fabaceae & Platymiscium floribundum Vogel $\ddagger$ & 3 & 0.097 & 3 & 0.1 & 0.1 & 0.2 & 0.41 \\
\hline Moraceae & Sorocea bonplandii (Baill.) W.C.Burger. et al. $\dagger$ & 3 & 0.095 & 3 & 0.1 & 0.1 & 0.2 & 0.41 \\
\hline Urticaceae & Cecropia pachystachya Trécul † & 4 & 0.075 & 3 & 0.1 & 0.1 & 0.2 & 0.41 \\
\hline Cyatheaceae & Alsophila setosa Kaulf. $\ddagger$ & 6 & 0.034 & 3 & 0.2 & 0.1 & 0.2 & 0.41 \\
\hline Euphorbiaceae & Pera glabrata (Schott) Poepp. ex Baill. $\ddagger$ & 3 & 0.089 & 3 & 0.1 & 0.1 & 0.2 & 0.40 \\
\hline Cannelaceae & Cinnamodendron dinisii Schwacke & 2 & 0.108 & 2 & 0.1 & 0.2 & 0.1 & 0.34 \\
\hline Rubiaceae & Amaioua guianensis Aubl. & 3 & 0.051 & 3 & 0.1 & 0.1 & 0.2 & 0.34 \\
\hline Myrtaceae & Neomitranthes glomerata (D.Legrand) D.Legrand † & 3 & 0.049 & 3 & 0.1 & 0.1 & 0.2 & 0.34 \\
\hline Myrsinaceae & Rapanea umbellata Mart. & 3 & 0.034 & 3 & 0.1 & 0.1 & 0.2 & 0.32 \\
\hline Myrtaceae & Eugenia sp. $3 \ddagger$ & 2 & 0.091 & 2 & 0.1 & 0.1 & 0.1 & 0.31 \\
\hline Moraceae & Brosimum cf. glaziovii Taub. $†$ & 2 & 0.090 & 2 & 0.1 & 0.1 & 0.1 & 0.31 \\
\hline Sapotaceae & Pouteria grandiflora (Wall.) Baehni $\dagger$ & 3 & 0.070 & 2 & 0.1 & 0.1 & 0.1 & 0.31 \\
\hline Bombacaceae & Spirotheca passifloroides Cuatrec. & 3 & 0.023 & 3 & 0.1 & 0.0 & 0.2 & 0.30 \\
\hline Salicaceae & Casearia decandra Jacq. & 3 & 0.019 & 3 & 0.1 & 0.0 & 0.2 & 0.30 \\
\hline Magnoliaceae & Talauma ovata A.St.-Hil. & 2 & 0.077 & 2 & 0.1 & 0.1 & 0.1 & 0.29 \\
\hline Fabaceae & Dalbergia frutescens (Vell.) Britton $\ddagger$ & 1 & 0.128 & 1 & 0.0 & 0.2 & 0.1 & 0.28 \\
\hline Picramniaceae & Picramnia gardneri subsp. gardneri Planch. $\doteqdot$ & 3 & 0.007 & 3 & 0.1 & 0.0 & 0.2 & 0.28 \\
\hline Sapindaceae & Matayba juglandifolia (Cambess.) Radlk. $\neq$ & 2 & 0.060 & 2 & 0.1 & 0.1 & 0.1 & 0.27 \\
\hline Rubiaceae & Coussarea contracta (Walp.) Müll.Arg. $\ddagger$ & 4 & 0.017 & 2 & 0.1 & 0.0 & 0.1 & 0.27 \\
\hline Myrtaceae & Eugenia sp. $2 \ddagger$ & 2 & 0.056 & 2 & 0.1 & 0.1 & 0.1 & 0.26 \\
\hline Rubiaceae & Simira cf. sampaioana (Standley) Steyerm. $\dagger$ & 2 & 0.048 & 2 & 0.1 & 0.1 & 0.1 & 0.25 \\
\hline Elaeocarpaceae & Sloanea monosperma Vell. & 2 & 0.033 & 2 & 0.1 & 0.1 & 0.1 & 0.23 \\
\hline Araliaceae & Oreopanax capitatus (Jacq.) Decne. \& Planch. $\ddagger$ & 1 & 0.091 & 1 & 0.0 & 0.1 & 0.1 & 0.22 \\
\hline Lauraceae & Ocotea aciphylla (Nees) Mez $\ddagger$ & 1 & 0.089 & 1 & 0.0 & 0.1 & 0.1 & 0.22 \\
\hline Myrtaceae & Gomidesia tijucensis (Kiaersk.) D.Legrand $\ddagger$ & 2 & 0.031 & 2 & 0.1 & 0.0 & 0.1 & 0.22 \\
\hline Sabiaceae & Meliosma sinuata Urb. $\dagger$ & 2 & 0.021 & 2 & 0.1 & 0.0 & 0.1 & 0.21 \\
\hline Boraginaceae & Cordia ecalyculata Vell. $\ddagger$ & 2 & 0.012 & 2 & 0.1 & 0.0 & 0.1 & 0.20 \\
\hline Myrtaceae & $\begin{array}{l}\text { Marlierea eugeniopsoides (D.Legrand \& Kaus.) } \\
\text { D.Legran. } \dagger\end{array}$ & 2 & 0.012 & 2 & 0.1 & 0.0 & 0.1 & 0.20 \\
\hline Celastraceae & Maytenus communis Reissek $\ddagger$ & 1 & 0.072 & 1 & 0.0 & 0.1 & 0.1 & 0.19 \\
\hline Moraceae & Ficus enormis (Mart. ex Miq.) Mart. $\ddagger$ & 1 & 0.070 & 1 & 0.0 & 0.1 & 0.1 & 0.19 \\
\hline Lauraceae & Ocotea tabacifolia (Meisn.) Rohwer $\dagger$ & 1 & 0.066 & 1 & 0.0 & 0.1 & 0.1 & 0.19 \\
\hline Fabaceae & Andira cf. ormosioides Benth. & 2 & 0.011 & 2 & 0.1 & 0.0 & 0.1 & 0.19 \\
\hline Melastomataceae & Henriettella glabra Cogn. $\dagger$ & 2 & 0.006 & 2 & 0.1 & 0.0 & 0.1 & 0.19 \\
\hline Lauraceae & Licaria armeniaca (Nees) Kosterm. $\dagger$ & 1 & 0.064 & 1 & 0.0 & 0.1 & 0.1 & 0.18 \\
\hline Lauraceae & Nectandra megapotamica (Spreng.) Mez $\dagger$ & 1 & 0.048 & 1 & 0.0 & 0.1 & 0.1 & 0.16 \\
\hline Caricaceae & Jacaratia spinosa (Aubl.) A.DC. $\dagger$ & 1 & 0.047 & 1 & 0.0 & 0.1 & 0.1 & 0.16 \\
\hline Fabaceae & Zollernia ilicifolia (Brongn.) Vogel $\ddagger$ & 1 & 0.034 & 1 & 0.0 & 0.1 & 0.1 & 0.14 \\
\hline Monimiaceae & Mollinedia triflora (Spreng.) Tul. $\ddagger$ & 2 & 0.015 & 1 & 0.1 & 0.0 & 0.1 & 0.14 \\
\hline Sapotaceae & Chrysophyllum viride Mart. \& Eichler $†$ & 2 & 0.011 & 1 & 0.1 & 0.0 & 0.1 & 0.14 \\
\hline Moraceae & Brosimum cf. guianense (Aubl.) Huber $\ddagger$ & 1 & 0.026 & 1 & 0.0 & 0.0 & 0.1 & 0.13 \\
\hline Fabaceae & Inga marginata Willd. $†$ & 2 & 0.009 & 1 & 0.1 & 0.0 & 0.1 & 0.13 \\
\hline Myrtaceae & Eugenia capitulifera O.Berg $\dagger$ & 1 & 0.022 & 1 & 0.0 & 0.0 & 0.1 & 0.12 \\
\hline Aquifoliaceae & Ilex amara (Vell). Loes. $\ddagger$ & 1 & 0.021 & 1 & 0.0 & 0.0 & 0.1 & 0.12 \\
\hline
\end{tabular}


continuation

\begin{tabular}{|c|c|c|c|c|c|c|c|c|}
\hline Family & Species & NI & BA & $\mathrm{Q}$ & $\mathrm{RD}$ & RDo & RF & $\mathrm{VI}$ \\
\hline Symplocaceae & Symplocos cf. tetrandra Mart. $\ddagger$ & 1 & 0.015 & 1 & 0.0 & 0.0 & 0.1 & 0.11 \\
\hline Cyatheaceae & Cyathea corcovadensis (Raddi) Domin $\ddagger$ & 1 & 0.010 & 1 & 0.0 & 0.0 & 0.1 & 0.10 \\
\hline Fabaceae & $\begin{array}{l}\text { Lonchocarpus cultratus (Vell.) A.M.G.Azevedo \& } \\
\text { H.C.Lima } \dagger\end{array}$ & 1 & 0.010 & 1 & 0.0 & 0.0 & 0.1 & 0.10 \\
\hline Myrtaceae & Plinia pauciflora M.L. Kawas. \& B.Holst $\dagger$ & 1 & 0.010 & 1 & 0.0 & 0.0 & 0.1 & 0.10 \\
\hline Fabaceae & Andira cf. fraxinifolia Benth. $\dagger$ & 1 & 0.009 & 1 & 0.0 & 0.0 & 0.1 & 0.10 \\
\hline Aquifoliaceae & Ilex theezans Mart. $\ddagger$ & 1 & 0.009 & 1 & 0.0 & 0.0 & 0.1 & 0.10 \\
\hline Rutaceae & Zanthoxylum rhoifolium Lam. $\ddagger$ & 1 & 0.009 & 1 & 0.0 & 0.0 & 0.1 & 0.10 \\
\hline Myristicaceae & Virola gardnerii (A.DC.) Warb. $\ddagger$ & 1 & 0.008 & 1 & 0.0 & 0.0 & 0.1 & 0.10 \\
\hline Lamiaceae & Vitex cf. polygama Cham. $\ddagger$ & 1 & 0.008 & 1 & 0.0 & 0.0 & 0.1 & 0.10 \\
\hline Anacardiaceae & Tapirira guianensis Aubl. $†$ & 1 & 0.007 & 1 & 0.0 & 0.0 & 0.1 & 0.10 \\
\hline Lauraceae & Nectandra membranacea (Sw.) Griseb. $\dagger$ & 1 & 0.005 & 1 & 0.0 & 0.0 & 0.1 & 0.10 \\
\hline Malpighiaceae & Bunchosia fluminensis Griseb. $†$ & 1 & 0.005 & 1 & 0.0 & 0.0 & 0.1 & 0.10 \\
\hline Rubiaceae & Randia nitida (Kunth) DC. $\dagger$ & 1 & 0.005 & 1 & 0.0 & 0.0 & 0.1 & 0.10 \\
\hline Myrtaceae & Myrceugenia reitzii D.Legrand \& Kausel $†$ & 1 & 0.005 & 1 & 0.0 & 0.0 & 0.1 & 0.10 \\
\hline Melastomataceae & Miconia holosericea (L.) DC. $\ddagger$ & 1 & 0.005 & 1 & 0.0 & 0.0 & 0.1 & 0.10 \\
\hline Erythroxylaceae & Erythroxylum ambiguum Peyr. $\dagger$ & 1 & 0.004 & 1 & 0.0 & 0.0 & 0.1 & 0.10 \\
\hline Piperaceae & Piper aduncum L. † & 1 & 0.004 & 1 & 0.0 & 0.0 & 0.1 & 0.10 \\
\hline Melastomataceae & Miconia tristis subsp. australis Wurdack $†$ & 1 & 0.004 & 1 & 0.0 & 0.0 & 0.1 & 0.10 \\
\hline Myrtaceae & Myrceugenia kleinii D.Legrand \& Kausel $†$ & 1 & 0.004 & 1 & 0.0 & 0.0 & 0.1 & 0.09 \\
\hline Melastomataceae & Miconia rigidiuscula Cogn. $\ddagger$ & 1 & 0.003 & 1 & 0.0 & 0.0 & 0.1 & 0.09 \\
\hline Celastraceae & Maytenus schumanniana Loes. $\ddagger$ & 1 & 0.003 & 1 & 0.0 & 0.0 & 0.1 & 0.09 \\
\hline Annonaceae & Rollinia cf. dolabripetala (Raddi) R.E.Fr. $\dagger$ & 1 & 0.002 & 1 & 0.0 & 0.0 & 0.1 & 0.09 \\
\hline Solanaceae & Solanum sp. $2 \dagger$ & 1 & 0.002 & 1 & 0.0 & 0.0 & 0.1 & 0.09 \\
\hline Solanaceae & Solanum sp. $1 \ddagger$ & 1 & 0.002 & 1 & 0.0 & 0.0 & 0.1 & 0.09 \\
\hline Salicaceae & Xylosma glaberrimum Sleumer $\dagger$ & 1 & 0.002 & 1 & 0.0 & 0.0 & 0.1 & 0.09 \\
\hline
\end{tabular}

of 0.444 for axis 1 and 0.145 for axis 2. Although some species were predominant in one of the plots, there was no great structural differentiation among them. Eugenia bocainensis, E. cuprea, Calyptranthes lanceolata,

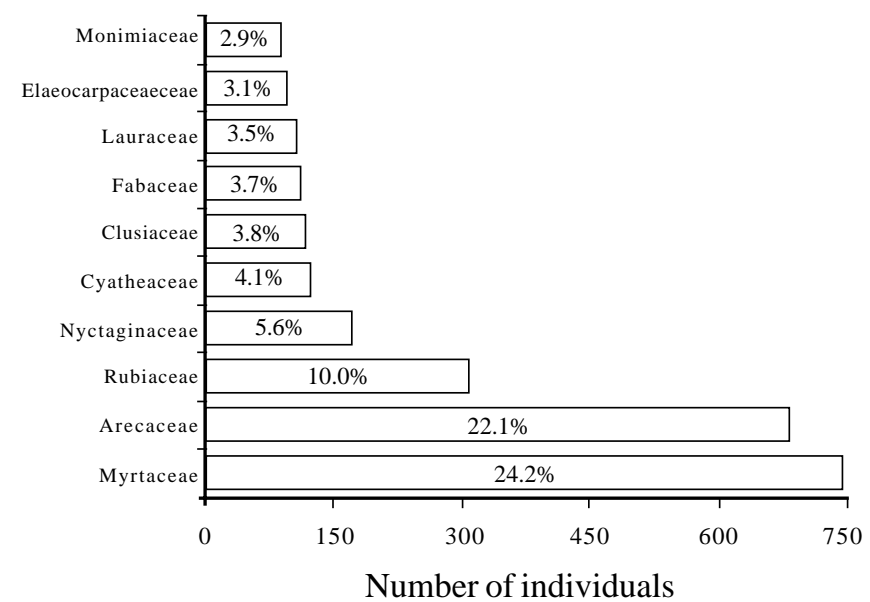

Figure 1. Distribution of the percentage of individuals by botanic family in the lowland Atlantic Rain Forest, Intervales State Park, Southeastern Brazil.
Ruprechtia laxiflora, Cyathea phalerata and Alsophila sternbergii were more abundant in Plot 1, and the latter was found exclusively in Plot 1 . The individuals of $A$. sternbergii were predominant in all four quadrats close to the Saibadela River (46 out of 64 individuals sampled), as shown in figure 2. Eugenia cambucarana also had 13 individuals exclusively sampled in the quadrats close to the river in Plot 1 . On the other hand, Psychotria mapoureoides, Mollinedia uleana, Calycorectes acutatus, Eugenia oblongata and Pilocarpus pauciflorus were more abundant in Plot 2. It is important to point out that Maytenus robusta and Eugenia subavenia, with 10 and 11 individuals respectively, were exclusively present in Plot 2.

Out of the 172 species found, 59 (34.3\%) presented a maximum of two individuals in the stand, wherein 41 of them were represented by only one individual (table 1). Moreover, only four out of the 18 species surveyed with two individuals had one individual per plot. The qualitative similarity between plots $\left(S_{J}=0.58\right)$ was outstandingly lower than the quantitative similarity 
Table 2. Comparisons of the number of individuals (NI) and basal area (BA) for 12 species with high value of importance (VI) sampled in two areas of lowland Atlantic Rain Forest, Intervales State Park, Southeastern Brazil. c: value of importance position of species in each plot.

\begin{tabular}{lrrrrrrr}
\hline \multirow{2}{*}{ Species } & \multicolumn{3}{c}{ Plot 1} & & \multicolumn{3}{c}{ Plot 2 } \\
\cline { 2 - 4 } \cline { 5 - 7 } & $\mathrm{c}$ & $\mathrm{NI}$ & $\mathrm{BA}$ & & $\mathrm{c}$ & $\mathrm{NI}$ & $\mathrm{BA}$ \\
\hline Euterpe edulis & 1 & 287 & 2.335 & & 1 & 384 & 2.601 \\
Sloanea guianensis & 4 & 45 & 2.226 & & 2 & 48 & 4.946 \\
Guapira opposita & 3 & 87 & 0.973 & 3 & 85 & 1.071 \\
Bathysa australis & 2 & 69 & 2.105 & & 9 & 31 & 0.673 \\
Eugenia mosenii & 6 & 56 & 0.455 & & 6 & 58 & 0.483 \\
Garcinia gardneriana & 14 & 28 & 0.276 & & 4 & 89 & 0.721 \\
Marlierea obscura & 7 & 44 & 0.621 & & 7 & 50 & 0.494 \\
Virola bicuhyba & 5 & 14 & 2.744 & 22 & 10 & 0.860 \\
Alchornea triplinervia & 9 & 11 & 1.924 & & 8 & 14 & 1.405 \\
Psychotria suterella & 25 & 21 & 0.096 & 5 & 74 & 0.315 \\
Marlierea tomentosa & 8 & 53 & 0.372 & & 24 & 25 & 0.144 \\
Mollinedia schottiana & 11 & 35 & 0.188 & & 16 & 31 & 0.134 \\
Total & & 750 & 14.316 & & 899 & 13.847 \\
\hline
\end{tabular}

$\left(S_{M}=0.94\right)$, due to the taxa found with greater abundance in the stand.

Vertical structure - The DCA analysis of species distribution throughout the height classes for all the species surveyed presented a gradient length of 20.99 and 10.15 and eingenvalues of 0.499 and 0.226 , for axes 1 and 2, respectively. The high eigenvalue of axis 1 showed a strong distribution of height classes in relation to the composition of species, forming three arbitrary groups (figure 3$)$ : stratum $A(\geq 26 \mathrm{~m})$, stratum $B(8 \mathrm{~m}<\mathrm{h}$ $<26 \mathrm{~m})$ and stratum $\mathrm{C}(\leq 8 \mathrm{~m})$. The stratum $\mathrm{A}$ was mainly distributed along the axis 2 due to the small number of individuals per species. These low densities in stratum A resulted in a lower floristic richness and diversity (table 3). Stratum B had higher basal area, richness and diversity of species. Stratum $C$ had higher density, however it concentrated many individuals in few species, in which 12 represented $67.9 \%$ of the total number of individuals, generating the lowest values of equability (table 3 ).

Table 4 displays the 12 species with the highest VI in each stratum. Euterpe edulis was the most abundant species in strata B and C, although it was more significantly associated with stratum C. Garcinia gardneriana and Eugenia mosenii were also wellrepresented in these two forest strata, although they did not present significantly higher density in any of the strata. Guapira opposita was more abundant in stratum $\mathrm{C}$ than in stratum B, where it showed a lower than expected number of individuals according to chi-square test $\left(\chi^{2}\right)$. The tree species significantly associated with stratum C were Psychotria suterella, Ixora burchelliana, Mollinedia schottiana, Marlierea

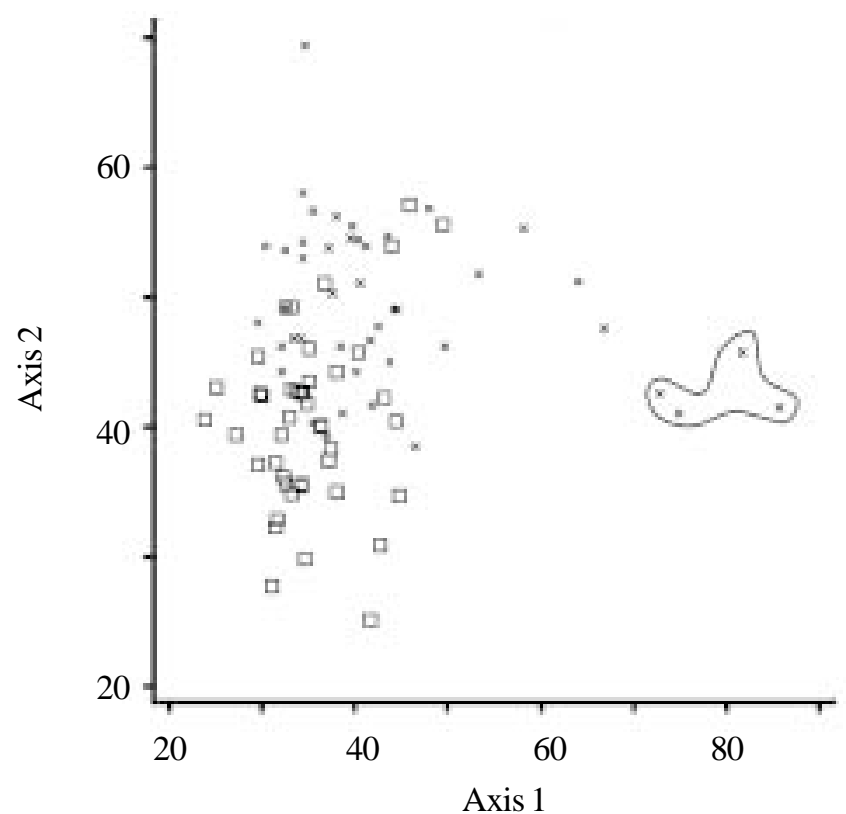

Figure 2. Ordination diagram produced by DCA, based on the distribution of the 48 most abundant species in 88 quadrats surveyed in the lowland Atlantic Rain Forest, Intervales State Park, Southeastern Brazil. The quadrats are classified for each sample plot. Quadrats in evidence are those closest to the Saibadela river in Plot 1. Eigenvalues (axis 1: 0.444; axis 2: 0.145). Plot $1=\times$, Plot $2=\square$. 


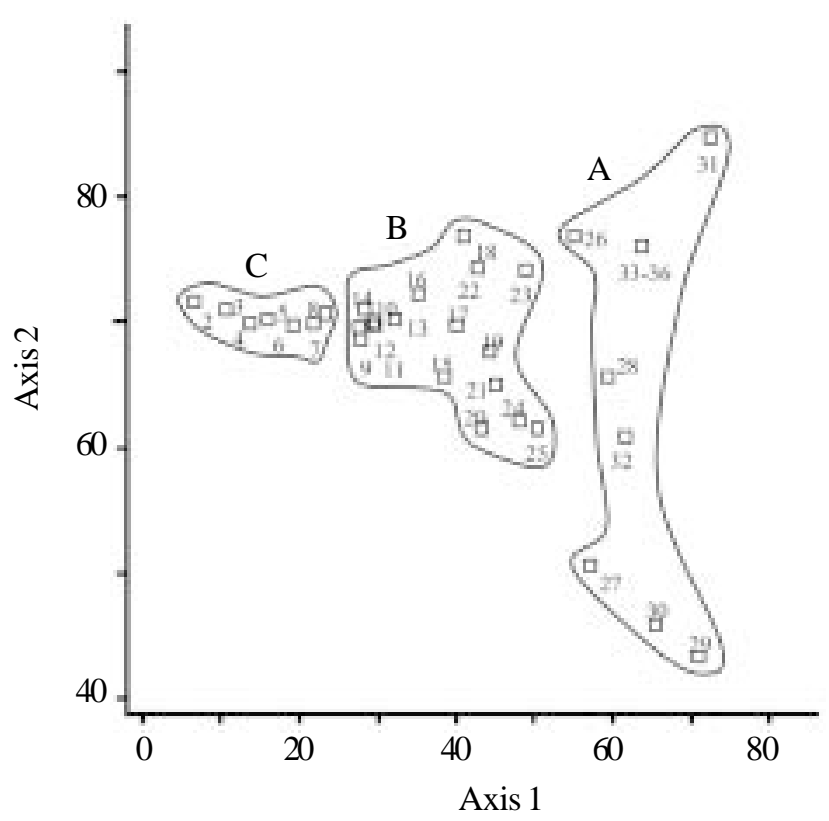

Figure 3. Ordination diagram of the height classes produced by DCA, based on estimated height of individuals of all species sampled in the stand of lowland Atlantic Rain Forest, Intervales State Park, Southeastern Brazil. The intervals of class are numbered in a sequence from 2 (individuals measuring up to $2 \mathrm{~m}$ height) to $33-36 \mathrm{~m}$ (individuals measuring from 33 to $36 \mathrm{~m}$ height). Eigenvalues (axis 1: 0.499; axis 2: 0.226). tomentosa, Eugenia cuprea, Alsophila sternbergii and Cyathea phalerata. The species significantly associated with stratum B were Bathysa australis, Marlierea obscura, Eugenia riedeliana, Tetrastylidium grandifolium and Swartzia flaemingii. Despite the fact that Alchornea triplinervia and especially Sloanea guianensis were included among the most important species in stratum B, both were significantly associated with stratum A, joined with

Table 3. Phytosociological data for the three vertical strata (A, B, and C) of lowland Atlantic Rain Forest, Intervales State Park, Southeastern Brazil.

\begin{tabular}{lrrr}
\hline Phytosociological data & \multicolumn{3}{c}{ Strata } \\
\cline { 2 - 4 } & \multicolumn{1}{c}{$\mathrm{A}$} & \multicolumn{1}{c}{$\mathrm{B}$} & \multicolumn{1}{c}{$\mathrm{C}$} \\
\hline Number of individuals & 103 & 1,356 & 1,556 \\
Basal area $\left(\mathrm{m}^{2}\right.$. ha $\left.^{-1}\right)$ & 12.298 & 18.066 & 3.910 \\
Height average (m) & 28.7 & 13.9 & 5.8 \\
Diameter average (cm) & 50.8 & 15.9 & 7.6 \\
Number of families & 19 & 45 & 41 \\
Number of species & 36 & 145 & 119 \\
Diversity index (H') & 3.172 & 3.895 & 3.440 \\
Equability index (J') & 0.885 & 0.782 & 0.720 \\
\hline
\end{tabular}

Table 4. Phytosociological descriptors for 12 tree species with high value of importance (VI) in each vertical stratum of lowland Atlantic Rain Forest, Intervales State Park, Southeastern Brazil. Qui-square $\left(\chi^{2}\right)$ tests the null hypothesis of the distribution of species abundance in each stratum $(\mathrm{A}, \mathrm{B}$, and $\mathrm{C})$ in relation to the total number of individuals in the stand. Values of $\chi^{2}$ followed by 'plus' (+) or 'minus' (-) indicate abundance above or below expected, respectively. $* P<0.05 ; * * P<0.01 ; * * * P$ $<0.001$; ns: not significant. $\S$ insufficient number of individuals for a qui-square test $\left(\chi^{2}\right)$.

\begin{tabular}{|c|c|c|c|c|c|c|c|c|}
\hline Species & NI & $\mathrm{BA}$ & $\mathrm{RD}$ & RDo & $\mathrm{RF}$ & VI & $\chi^{2}$ & $P$ \\
\hline \multicolumn{9}{|l|}{ Stratum A } \\
\hline Sloanea guianensis & 16 & 4.610 & 15.5 & 18.9 & 14.1 & 48.6 & +47.80 & $* * *$ \\
\hline Virola bicuhyba & 10 & 3.184 & 9.7 & 13.1 & 10.1 & 32.9 & +91.89 & $* * *$ \\
\hline Hyeronima alchorneoides & 8 & 1.566 & 7.8 & 6.4 & 7.1 & 21.3 & +82.44 & $* * *$ \\
\hline Alchornea triplinervia & 7 & 1.349 & 6.8 & 5.5 & 6.1 & 18.4 & +37.32 & $* * *$ \\
\hline Pseudopiptadenia warmingii & 6 & 1.029 & 5.8 & 4.2 & 6.1 & 16.1 & +87.69 & $* * *$ \\
\hline Callisthene cf. dryadum & 4 & 1.505 & 3.9 & 6.1 & 4.0 & 14.1 & $\S$ & - \\
\hline Pterocarpus rohrii & 6 & 0.433 & 5.8 & 1.8 & 6.1 & 13.7 & +31.88 & $* * *$ \\
\hline Copaifera trapezifolia & 4 & 1.152 & 3.9 & 4.7 & 4.0 & 12.7 & $\S$ & - \\
\hline Cariniana estrellensis & 3 & 1.143 & 2.9 & 4.7 & 3.0 & 10.6 & $\S$ & - \\
\hline Aspidosperma cf. compactinervium & 2 & 1.063 & 1.9 & 4.7 & 2.0 & 8.3 & $\S$ & - \\
\hline Schizolobium parahyba & 2 & 0.864 & 1.9 & 3.6 & 2.0 & 7.5 & $\S$ & - \\
\hline Ficus gomelleira & 1 & 1.325 & 1.0 & 5.4 & 1.0 & 7.4 & $\S$ & - \\
\hline \multicolumn{9}{|l|}{ Stratum B } \\
\hline Euterpe edulis & 249 & 2.550 & 7.1 & 7.8 & 18.4 & 33.3 & 2.10 & $\mathrm{~ns}$ \\
\hline Bathysa australis & 78 & 2.603 & 7.3 & 4.7 & 5.8 & 17.7 & +23.52 & $* * *$ \\
\hline
\end{tabular}




\begin{tabular}{|c|c|c|c|c|c|c|c|c|}
\hline Species & $\mathrm{NI}$ & $\mathrm{BA}$ & $\mathrm{RD}$ & RDo & $\mathrm{RF}$ & VI & $\chi^{2}$ & $P$ \\
\hline \multicolumn{9}{|l|}{ Stratum B (cont.) } \\
\hline Sloanea guianensis & 60 & 2.407 & 6.7 & 4.5 & 4.4 & 15.6 & +7.47 & $* *$ \\
\hline Marlierea obscura & 70 & 1.014 & 2.8 & 4.8 & 5.2 & 12.8 & +17.53 & $* * *$ \\
\hline Guapira opposita & 55 & 1.460 & 4.1 & 4.0 & 4.1 & 12.1 & -6.18 & $*$ \\
\hline Garcinia gardneriana & 57 & 0.720 & 2.0 & 3.8 & 4.2 & 10.0 & 0.29 & $\mathrm{~ns}$ \\
\hline Eugenia mosenii & 46 & 0.547 & 1.5 & 3.7 & 3.4 & 8.6 & 0.44 & $\mathrm{~ns}$ \\
\hline Alchornea triplinervia & 16 & 1.850 & 5.2 & 1.6 & 1.2 & 8.0 & 1.61 & $\mathrm{~ns}$ \\
\hline Tetrastilidium grandifolium & 36 & 0.733 & 2.1 & 2.4 & 2.7 & 7.1 & +10.60 & $* *$ \\
\hline Eugenia riedeliana & 25 & 1.103 & 3.1 & 2.1 & 1.8 & 7.1 & +5.55 & $*$ \\
\hline Swartzia flaemingii & 18 & 0.967 & 2.7 & 1.8 & 1.3 & 5.9 & +4.95 & $*$ \\
\hline Calycorectes australis & 22 & 0.808 & 2.3 & 1.9 & 1.6 & 5.8 & 0.51 & $\mathrm{~ns}$ \\
\hline \multicolumn{9}{|l|}{ Stratum C } \\
\hline Euterpe edulis & 359 & 1.645 & 23.1 & 21.2 & 8.98 & 53.3 & +6.73 & $* *$ \\
\hline Guapira opposita & 117 & 0.584 & 7.5 & 7.5 & 6.82 & 21.9 & +8.66 & $* *$ \\
\hline Psychotria suterella & 95 & 0.411 & 6.1 & 5.3 & 5.19 & 16.6 & +42.17 & $* * *$ \\
\hline Eugenia mosenii & 68 & 0.392 & 4.4 & 5.1 & 4.87 & 14.3 & 1.28 & $\mathrm{~ns}$ \\
\hline Mollinedia schottiana & 63 & 0.295 & 4.1 & 3.8 & 4.65 & 12.5 & +23.74 & $* * *$ \\
\hline Marlierea tomentosa & 62 & 0.356 & 4.0 & 4.6 & 3.8 & 12.4 & +11.21 & $* * *$ \\
\hline Alsophila sternbergii & 59 & 0.534 & 3.8 & 6.9 & 1.1 & 11.8 & +19.64 & $* * *$ \\
\hline Garcinia gardneriana & 60 & 0.278 & 3.9 & 3.6 & 3.9 & 11.3 & 0.00 & $\mathrm{~ns}$ \\
\hline Cyathea phalerata & 54 & 0.286 & 3.5 & 3.7 & 3.4 & 10.5 & +23.57 & $* * *$ \\
\hline Ixora burchelliana & 47 & 0.160 & 3.0 & 2.1 & 3.7 & 8.8 & +20.40 & $* * *$ \\
\hline Eugenia cuprea & 45 & 0.190 & 2.9 & 2.5 & 3.4 & 8.7 & +12.56 & $* * *$ \\
\hline Ocotea teleiandra & 27 & 0.098 & 1.7 & 1.3 & 2.4 & 5.4 & 2.02 & $\mathrm{~ns}$ \\
\hline
\end{tabular}

Virola bicuhyba, Hyeronima alchorneoides, Pseudopiptadenia warmingii and Pterocarpus rohrii.

\section{Discussion}

Horizontal structure - The six families with the largest number of species found in this study presented the greatest floristic richness in previous studies of the Atlantic Rain Forest at Serra do Mar, Ubatuba (Silva \& Leitão Filho 1982, Sanches et al. 1999), and the hill slopes of Ilha do Cardoso, Cananéia (Melo \& Mantovani 1994). This confirms their importance in the general characterization of the Atlantic Rain Forest. Myrtaceae was the family with the largest number of species in this study, with the genus Eugenia alone represented by 19 species. This great richness of Myrtaceae, especially the genus Eugenia, corroborates the pattern found for the Atlantic Rain Forest in São Paulo State (Scudeller et al. 2001) and in Brazil (Oliveira Filho \& Fontes 2000). According to Mori et al. (1983) and Peixoto \& Gentry (1990), the Myrtaceae family has great ecological importance on the entire Brazilian coast due to its great abundance and species richness.
Arecaceae followed Myrtaceae in number of individuals, as previously recorded by Melo et al. (1998) in Juréia and by Sanches et al. (1999) in Ubatuba. The Arecaceae family, although represented by only two species in the present study, had great influence on the structure of the studied tree community, due mostly to the high abundance of Euterpe edulis. Sztutman \& Rodrigues (2002) found the same pattern for another forest on the south of São Paulo State. At Base Saibadela, E. edulis occurred abundantly in several distinct environmental conditions. This pattern may be linked to the great investment and success in the reproduction of the species, as reported by Reis \& Kageyama (2000). The importance of E. edulis in the Atlantic Rain Forest is due also to the great availability of fruits produced annually, which can reach up to 377.000 ha $^{-1}$ year-1 $^{-1}$ (Reis \& Kageyama 2000). They serve as a main source of food to many mammals and large birds (Galetti \& Chivers 1995, Galetti et al. 1997).

Despite the high abundance of Euterpe edulis (see also Scudeller et al. 2001), its genetic diversity has been seriously threatened by clandestine exploration of palmhearts (Reis \& Reis 2000). In our study, harvested palms 
had an average ( \pm SD) diameter at breast height (DBH) of $12.15 \mathrm{~cm} \pm 1.53$, larger than $\mathrm{DBH}$ of the living individuals $(9.04 \mathrm{~cm} \pm 2.96)$, showing that the largest individuals were the favorite to be harvested by the clandestine collectors.

Diversity, floristic, and structural comparisons Phytosociological surveys conducted in the Atlantic Rain Forest in the São Paulo State often showed tree diversity indexes ranging from 4.07 to 4.31 nat.ind. ${ }^{-1}$ (Silva \& Leitão Filho 1982, Mantovani et al. 1990, Leitão Filho 1993, Melo et al. 1998). The lowest diversity index $\left(\mathrm{H}^{\prime}=3.85\right.$ nat.ind. $\left.^{-1}\right)$ found here may have been the result of the method employed, which restricted the number of ecotones within the stand, and consequently reduced the environmental heterogeinity of the forest mosaic which composes the Atlantic Rain Forest.

Some species presented different abundance between the two sample plots, especially in quadrats associated with the Saibadela rivercourse and the permanent rise of the water table in Plot 1, where the soil is poorly drained. These factors generate greater floristic and structural differentiation of these sites compared to the rest of the stand. Therefore, the short gradients shown by DCA, may possibly derive from variations occurred in the ordination of the main species, which was small in both plots, resulting in great quantitative similarity between them. On the other hand, in terms of qualitative similarity, the floristic differences between the plots were high. Such floristic distinction results from the large amount of little abundant taxa found at the Base Saibadela, confirming what had already been observed in previous studies, that the great richness results in great diversity of the tree species in the Atlantic Rain Forest (Silva \& Leitão Filho 1982, Mantovani et al. 1990, Melo et al. 1998, Sanches et al. 1999).

Vertical Structure - Souza et al. (2003) argued that the cluster and discriminant multivariate analyses, are viable to assess the vertical tree structure in a Semideciduous Seasonal Forest where three levels of distribution are found. In the present study, although the DCA is an indirect method, it generated both a gradient of height intervals and a trend of occurrence of three arbitrary strata in the sampled area. Thus, we argue then that A is the canopy stratum, composed by emerging individuals; $\mathrm{B}$ is the subcanopy stratum, composed by large to medium-size trees and representatives of stratum A; and $\mathrm{C}$ is the understorey stratum, composed by small individuals and immature representatives from strata $\mathrm{A}$ and $\mathrm{B}$.

Stratum B showed the highest floristic richness and diversity of the three strata considered in this study. As argued by Terborgh (1992), tree diversity must be higher in the intermediate levels in tropical forests, where trees are more susceptible to a great variety of light conditions due to shadowing provided by the various shapes of crowns of emerging trees and the canopy, and by gaps created in the forest.

Euterpe edulis and Garcinia gardneriana, besides several species of Myrtaceae, were expressively represented in strata B and $\mathrm{C}$ in the sample plots. Guapira opposita (Nyctaginaceae), which is abundant and highly spread throughout the Atlantic Rain Forest (Mantovani et al. 1990, Leitão Filho 1993, Scudeller et al. 2001), was also among the most important species in strata $\mathrm{B}$ and $\mathrm{C}$, and the most abundant species in the understorey of the stand. Despite being seldom discussed in literature, other taxa are very characteristics of understorey. As observed in the present study, stratum $\mathrm{C}$ was well represented by the Cyatheaceae, with species such as Alsophila sternbergii and Cyathea phalerata, and by the Rubiaceae, with Psychotria suterella, Ixora burchelliana and Rudgea jasminoides, besides other immature individuals, e.g. Bathysa australis.

Terborgh (1992) argued that in mature tropical forests, the canopy and emerging tree component present a smaller number of individuals and a smaller floristic richness, as seen in this study. Nevertheless, the Fabaceae family was well represented in stratum A of the stand, showing the greatest richness ( 8 species) and density (22 individuals). Besides the Fabaceae, other important species in this stratum were Virola bicuhyba, Hyeronima alchorneoides, Sloanea guianensis and Alchornea triplinervia. As reported by Sztutman \& Rodrigues (2002), S. guianensis and A. triplinervia have large size representatives occurring respectively in well-drained soils of the Atlantic Rain Forest and in the Costal Plain or Restinga Forest located in PariqueraAçu, SP. It is possible to assert that these species are peculiar of the canopy in the Atlantic Rain Forest which can occasionally appear as emergent trees.

The method herein applied disregard the mosaic character in which tropical forests are in general organized, where there are spots of different ages, compositions and structures (Whitmore 1975), in which the vertical distribution varies. Moreover, such distribution in tropical forests is not always evident and although the limits may be imperceptible along the stratification, Richards (1996) argues that for descriptive purposes it is convenient to consider the existence of three strata (A, B and C). Therefore we recommended that the method used in the present study be applied to other 
types of forests whose stratification is poorly defined, aiming to test the effectiveness of the DCA analysis in these situations. More refined discussions with regard to the definition of strata, and the ecology of the occupation by plants and animals along stratification, can result from such investigations. Thus, the study of vertical structure, so little investigated up to now, must be very important, maybe more than those related with the horizontal structure, considering that the difference of occupation of niches in different strata is one of the most crucial factors to explain the high diversity in tropical forests (Terborgh 1992).

Acknowledgements - The authors would like to express their gratitude to M.A. Pizo, F.R. Scarano and the anonymous reviewers for his critical reading of the manuscript. Special thanks to V.B. Zipparro and Sr. Elias for field assistance; to M. Sobral and M.L. Kawasaki (Myrtaceae), V.A.O. Dittrich (Cyatheaceae) and P.L.R. Moraes (Lauraceae) for the plant identification; to A. Barbosa for helping English review, to Instituto Florestal and Fundação Florestal, for permission to work at Intervales State Park and particularly Jeanette, for logistic support; Fapesp (proc. n. 95/9626-0) for financial support; $\mathrm{CNPq}$ for the grant and research productivity fellowships for L.P.C. Morellato and to Capes for the fellowship to F.A.G. Guilherme.

\section{Literature cited}

ALMEIDA, F.F.M. 1974. Fundamentos geológicos do relevo paulista. Série Teses e Monografias - 14, USP, São Paulo.

APG (ANGIOSPERM PHYLOGENY GROUP). 2003. An update of the angiosperm phylogeny group classification for the orders and families of flowering plants: APG II. Botanical Journal of the Linnean Society 141:399-436.

BROWER, J.E. \&ZAR, J.H. 1984. Field and laboratory methods for general ecology. Wm. C. Brown Pub., Dubuque.

CAUSTON, D.R. 1988. An introduction to vegetation analysis, principles and interpretation. Unwin Hyman, London.

DIETZ, L.A. 2001. Intervales, modelo para a conservação florestal. In Intervales: fundação para a conservação e a produção florestal do estado de São Paulo. Secretaria do Meio Ambiente, Fundação Florestal, São Paulo, p.203-207.

GALETTI, M. \& CHIVERS, D.J. 1995. Palm harvest threatens Brazil's best protected area of Atlantic forest. Oryx 29:225-226.

GALETTI, M., MARTUSCELLI, P., OLMOS, F. \& ALEIXO, A. 1997. Ecology and conservation of the jacutinga Pipile jacutinga in the Atlantic forest of Brazil. Biological Conservation 82:31-39.

HILL, M.O. \& GAUCH, H.G. 1980. Detrended correspondence analysis: an improved ordination technique. Vegetatio 42:47-58.
IVANAUSKAS, N.M., MONTEIRO, R. \& RODRIGUES, R.R. 2000. Similaridade florística entre áreas de floresta Atlântica no estado de São Paulo. Brazilian Journal of Ecology 1/2:71-81.

KERSTEN, R.A. \& SILVA, S.M. 2001. Composição florística e distribuição espacial de epífitas vasculares em floresta de planície litorânea da Ilha do Mel, Paraná, Brasil. Revista Brasileira de Botânica 24:213-226.

KERSTEN, R.A. \& SILVA, S.M. 2002. Composição florística e estrutura do componente epifítico vascular em floresta ombrófila mista aluvial do Rio Barigüi, Paraná, Brasil. Revista Brasileira de Botânica 25:259-267.

KREBS, C.J. 1999. Ecological methodology. Addison Wesley Longman, Menlo Park.

LATHAM, P.A., ZUURING, H.R. \& COBLE, D.W. 1998. A method for quantifying vertical forest structure. Forest Ecology and Management 104:157-170.

LEITÃO FILHO, H.F. 1987. Considerações sobre a florística de florestas tropicais e sub-tropicais do Brasil. IPEF 35:41-46.

LEITÃO FILHO, H.F. (org.). 1993. Ecologia da Mata Atlântica em Cubatão. Unesp/Unicamp, São Paulo/Campinas.

LEITÃO FILHO, H.F. 1994. Diversity of arboreal species in Atlantic rain forest. Anais da Academia Brasileira de Ciências 66:91-96.

MANTOVANI, W. 1998. Dinâmica da Floresta Pluvial Atlântica. In Anais do IV Simpósio de Ecossistemas Brasileiros, (S. Watanabe, ed.). Academia de Ciências do Estado de São Paulo, São Paulo, p.1-20.

MANTOVANI, W., RODRIGUES, R.R., ROSSI, L., ROMANIUC NETO, S., CATHARINO, E.L.M. \& CORDEIRO, I. 1990. A vegetação da Serra do Mar em Salesópolis, SP. In Anais do II Simpósio de Ecossistemas da Costa Sul e Sudeste Brasileira, (S. Watanabe, ed.). Academia de Ciências do Estado de São Paulo, São Paulo, p.348-384.

MELO, M.M.R.F. \& MANTOVANI, W. 1994. Composição florística e estrutura de trecho de Mata Atlântica de encosta, na Ilha do Cardoso (Cananéia, SP, Brasil). Boletim do Instituto de Botânica 9:107-158.

MELO, M.M.R.F., OLIVEIRA, R.J., ROSSI, L., MAMEDE, M.C.H. \& CORDEIRO, I. 1998. Fitossociologia de um trecho de Mata Atlântica na planície do rio Verde, Estação Ecológica da Juréia-Itatins, SP, Brasil. In Anais do IV Simpósio de Ecossistemas Brasileiros (S. Watanabe, ed.) Academia de Ciências do Estado de São Paulo, São Paulo, p.49-56.

MOFFETT, M.W. 2000. What's "up"? A critical look at the basic terms of canopy biology. Biotopica 32:569-596.

MORELLATO, L.P.C., TALORA, D.C., TAKAHASI, A., BENCKE, C.C., ROMERA, E.C. \& ZIPPARRO, V.B. 2000. Phenology of Atlantic Rain Forest trees: a comparative study. Biotropica 32:811-823.

MORI, S.A., BOOM. B.M. \& PRANCE, G.T. 1981. Distribution patterns and conservation of eastern Brazilian coastal forest tree species. Brittonia 33:233-245. 
MORI, S.A., BOOM, B.M., CARVALHO, A.M. \& SANTOS, T.S. 1983. Ecological importance of Myrtaceae in an Eastern Brazilian wet forest. Biotropica 15:68-70.

MUELLER-DOMBOIS, D. \& ELLENBERG, H. 1974. Aims and methods for vegetation ecology. John Wiley \& Sons, New York.

MYERS, N., MITTERMEIER, R.A., MITTERMEIER, C.G., FONSECA, G.A.B. \& KENT, J. 2000. Biodiversity hotspots for conservation priorities. Nature 403:853-858.

OLIVEIRA, R.J., MANTOVANI, W. \& MELO, M.M.R.F. 2001. Estrutura do componente arbustivo-arbóreo da floresta atlântica de encosta, Peruíbe, SP. Acta Botanica Brasilica 15:391-412.

OLIVEIRA FILHO, A.T. \& FONTES, M.A.L. 2000. Patterns of floristic differentiation among Atlantic Forests in Southeastern Brazil, and the influence of climate. Biotropica 32:793-810.

PEIXOTO, A.L. \& GENTRY, A.H. 1990. Diversidade e composição florística da mata de tabuleiro na Reserva Florestal de Linhares (Espírito Santo, Brasil). Revista Brasileira de Botânica 13:19-25.

REIS, A. \& KAGEYAMA, P.Y. 2000. Dispersão de sementes do palmiteiro (Euterpe edulis Martius - Palmae). In Euterpe edulis Martius - palmiteiro: biologia, conservação e manejo (M.S. Reis \& A. Reis, eds.). Herbário Barbosa Rodrigues, Itajaí, p.60-92.

REIS, M.S. \& REIS, A. 2000. Euterpe edulis Martius palmiteiro: biologia, conservação e manejo. Herbário Barbosa Rodrigues, Itajaí.

RICHARDS, P.W. 1996. The tropical rain forest: an ecological study. Cambridge University Press, Cambridge.

ROTH, I. 1987. Stratification of a tropical forest as seen in dispersal types. Dr W. Junk Publishers, Dordrecht.
SANCHES, M., PEDRONI, F., LEITÃO FILHO, H.F. \& CÉSAR, O. 1999. Composição florística de um trecho de floresta ripária na Mata Atlântica em Picinguaba, Ubatuba, SP. Revista Brasileira de Botânica 22:31-42.

SCUDELLER, V.V., MARTINS, F.R. \& SHEPHERD, G.J. 2001. Distribution and abundance of arboreal species in the atlantic ombrophilous dense forest in Southeastern Brazil. Plant Ecology 152:185-199.

SETZER, J. 1966. Atlas climático e ecológico do Estado de São Paulo. Comissão Interestadual da Bacia ParanáUruguai/Cesp, São Paulo.

SHEPHERD, G.J. 1994. FITOPAC I. Manual do usuário. Unicamp, Campinas.

SILVA, A.F. \& LEITÃO FILHO, H.F. 1982. Composição florística e estrutura de um trecho de Mata Atlântica de encosta no município de Ubatuba (São Paulo, Brasil). Revista Brasileira de Botânica 5:43-52.

SOUZA, D.R., SOUZA, A.L. GAMA, J.R.V. \& LEITE, H.G. 2003. Emprego de análise multivariada para estratificação vertical de florestas ineqüiâneas. Revista Árvore 27:59-63.

SZTUTMAN, M. \& RODRIGUES, R.R. 2002. O mosaico vegetacional numa área de floresta contínua de planície litorânea, Parque Estadual da Campina do Encantado, Pariquera-Açu, SP. Revista Brasileira de Botânica 25:161-176.

TERBORGH, J. 1992. Diversity and the tropical rain forest. Scientific American Library, New York.

WHITMORE, T.H. 1975. Tropical rain forest of the far east. Claredon Press, Oxford.

ZAR, J.H. 1996. Biostatistical analysis. Prentice Hall, New Jersey. 